\title{
Calibration of Raman Lidar Water Vapor Mixing Ratio Measurements Using Zenithal Measurements of Diffuse Sunlight and a Radiative Transfer Model
}

\author{
Constantino Muñoz-Porcar ${ }^{\circledR}$, Adolfo Comerón, Michaël Sicard, Ruben Barragan, David Garcia-Vizcaino, \\ Alejandro Rodríguez-Gómez, Francesc Rocadenbosch ${ }^{\circledR}$, and María José Granados-Muñoz
}

\begin{abstract}
Among the different techniques available for measuring the atmospheric water vapor content, Raman lidars stand out as accurate instruments providing detailed profiles with high temporal and altitude resolution. Their principle is based on obtaining the range-resolved ratio of the lidar signals corresponding to Raman returns from water vapor and nitrogen molecules, which is proportional to the water vapor mixing ratio. To do this, it is necessary to determine a calibration factor, specific of each lidar instrument. A method for obtaining this parameter, based on zenith measurements of diffuse sunlight, on Raman scattering models and on simulations, using a radiative transfer model, to estimate sky radiances at the wavelengths of interest, has been applied to the lidar system of Universitat Politècnica de Catalunya (UPC; Technical University of Catalonia, Barcelona, Spain). A set of calibrations, performed between 2016 and 2017, has permitted assessing the calibration procedure and analyzing the stability of the calibration factor in the UPC instrument. Results show that although the calibration factor can remain stable for long periods of time, it can suffer sudden variations that make indispensable to implement a convenient and reliable procedure to perform regular calibrations. We show that the method, which can be applied to any lidar with water vapor and nitrogen Raman channels, can completely dispense with radiosonde data. The calibration method is validated by
\end{abstract}

Manuscript received December 21, 2017; revised February 23, 2018, May 7, 2018, and June 5, 2018; accepted June 6, 2018. This work was supported in part by the European Union H2020 Programme through the ACTRIS-2 Project under Grant GA 654109, in part by the Spanish Ministry of Economy and Competitiveness-European Regional Development Funds under Grant TEC2015-63832-P, in part by Generalitat de Catalunya (Grup de Recerca Consolidat) under Grant 2014-SGR-583, in part by the Unidad de Excelencia Maria de Maeztu through the Agencia Estatal de Investigación, Spain, under Grant MDM-2016-0600, and in part by the Juan de la CiervaFormación Program under Grant FJCI-2015-23904. (Corresponding author: Constantino Muñoz-Porcar.)

C. Muñoz-Porcar, A. Comerón, D. Garcia-Vizcaino, A. Rodríguez-Gómez, and M. J. Granados-Muñoz are with the CommSensLab, Department of Signal Theory and Communications, Universitat Politècnica de Catalunya (BarcelonaTech-UPC), 08034 Barcelona, Spain (e-mail: constan@ tsc.upc.edu; comeron@tsc.upc.edu; dgarcia@tsc.upc.edu; alejandro@ tsc.upc.edu; maria.jose.granados@tsc.upc.edu).

M. Sicard, R. Barragan, and F. Rocadenbosch are with the CommSensLab, Department of Signal Theory and Communications, Universitat Politècnica de Catalunya (BarcelonaTech-UPC), 08034 Barcelona, Spain, and also with the CTE-CRAE-Grup de Recerca en Ciències i Tecnologies de l'Espai, Universitat Politècnica de Catalunya, 08034 Barcelona, Spain (e-mail: sicard@tsc.upc.edu; ruben.barragan@tsc.upc.edu; roca@tsc.upc.edu).

Color versions of one or more of the figures in this paper are available online at http://ieeexplore.ieee.org.

Digital Object Identifier 10.1109/TGRS.2018.2851064 comparison with simultaneous radiosonde water vapor measurements. Limitations of radiosondes for validating - and eventually calibrating-water vapor Raman lidars have been revealed.

Index Terms-Calibration, lidar, Raman, water vapor.

\section{INTRODUCTION}

W ATER vapor is one of the most important constituents in the earth's atmosphere. It plays a key role in the global radiative budget and in energy transport mechanisms and is one of the main contributors to the greenhouse effect [1]. Moreover, it affects the cloud formation and microphysics and modifies aerosol particle properties through hygroscopic growth [2], thus indirectly contributing to changes in the atmospheric radiative forcing.

Because of water vapor temporal and spatial variability, highly resolved observations are the key to gain insight into its role on earth's climate. Balloon-borne radiosondes are widely used for water vapor measurements. Despite providing accurate measurements with large vertical and spatial coverage, they present the major drawbacks of a low temporal resolution (one or two launches per day) and elevated cost [3]. Furthermore, water vapor measurement techniques include satellites, microwave radiometry [4], [5], lidar, sun photometry [6], star photometry [7], DIAL [8], and infrared [9] spectrometry or GPS [10].

Raman lidars have emerged in the last decades as a powerful tool for providing detailed water vapor profiles with high vertical and temporal resolutions [11]-[13]. The water vapor Raman lidar technique consists in obtaining the range-resolved ratio of rotational-vibrational Raman scattering intensities from water vapor and nitrogen molecules, which is proportional to the water vapor mixing ratio (WVMR) [14]. To do this, a calibration factor specific of each lidar instrument has to be determined (Section II). Typical methods to derive this factor are based on comparisons with simultaneous co-located measurements from reference instruments, such as radiosondes [13], [14], providing water vapor profiles, or microwave radiometers [4], [5], and measuring the column-integrated water vapor content. Other methods use calibrated sources of light with known spectral features to determine the overall system transmission at both Raman wavelengths [15], [16]. 
In 1999, Sherlock et al. [17] described an alternative method for obtaining the calibration factor of a Raman water vapor lidar that is not dependent on other reference instruments nor on calibrated sources of light. Their method has been applied to the lidar system of Universitat Politècnica de Catalunya (UPC; Technical University of Catalonia, Catalonia, Spain), which is fully described in [18]. The method relies on zenith lidar measurements of diffuse sunlight, on simulations using a radiative transfer model (GAME) [19] to determine sky radiances at water vapor and nitrogen Raman wavelengths and on calculations involving well established Raman scattering models [20]-[22] and system elements data (Section III). Sherlock et al. [17] found that independent aerosol measurements are essential for constraining the parameters of the radiative transfer model and reducing the uncertainty of the calibration. When they applied the method to the Observatoire de Haute Provence Raman lidar system in 1999, they could not have available such information. In our case, simultaneous sun photometer data and aerosol lidar products have been used as inputs of GAME. Other auxiliary measurements required by the model include profiles of meteorological variables, which can be obtained either from radiosonde data or from surface measurements and atmospheric models. The latest option has been our choice in order to define a calibration procedure dependent uniquely on auxiliary instrumentation run at the same lidar station. Calibrations using this method were performed between 2016 and 2017 under different meteorological conditions, and the stability of calibration factors was analyzed (Section IV). Comparisons between lidar water vapor measurements using these calibrations and available radiosonde data have been used to validate the results of the method (Section V). Some limitations and uncertainties associated with the use of radiosondes as reference instruments have in turn been revealed.

\section{WATER VAPOR Mixing RATiO FROM LIDAR SIGNALS}

The atmospheric WVMR is defined as the ratio between the water vapor mass in a given volume and the mass of the rest of the volume. As the water vapor mass is always much lesser than the mass of the rest of molecules in the volume, the latter can be approximated by the mass of dry air that occupies the same volume. Taking into account, moreover, that the molecular weight of water molecules is $18 \mathrm{~g} / \mathrm{mol}$, that the mean molecular weight of the air is $28.96 \mathrm{~g} / \mathrm{mol} \mathrm{[23]} \mathrm{and}$ that the nitrogen $\left(N_{2}\right)$ volume proportion in the air (hence the proportion of its molecule number concentration) is $78.08 \%$, the mixing ratio, $\omega$, can be expressed as

$$
\omega=\frac{0.7808 \times 18 \times N_{W}}{28.96 \times N_{N}}
$$

where $N_{W}$ is the number concentration of water vapor molecules and $N_{N}$ the number concentration of nitrogen molecules. The detected signal voltage (or the count rate per bin) from a given range $R$ in the nitrogen and water vapor Raman channels of a lidar instrument, once "dark-current" offset and diffuse sky radiation have been subtracted, is given by the lidar equation

$$
\begin{aligned}
S_{X}(R)=\frac{E A_{r} c K_{X} O_{X}(R) N_{X}(R) \frac{\partial \sigma_{\text {Xeff }}}{\partial \Omega}}{2 R^{2}} \\
\quad \times \exp \left\{-\int_{0}^{R}\left[\alpha\left(r, \lambda_{0}\right)+\alpha\left(r, \lambda_{X}\right)\right] d r\right\}
\end{aligned}
$$

where $X$ is either $W$ or $N$ (referring, respectively, to water vapor and nitrogen channels), $E$ is the emitted pulse energy [J], $\lambda_{0}$ is the emitted wavelength [m], $A_{r}$ is the effective receiving area $\left[\mathrm{m}^{2}\right], c$ is the speed of light $\left[\mathrm{ms}^{-1}\right], K_{X}[\mathrm{~N} / \mathrm{W}]$ is a system constant that takes into account the end-to-end transmittance of the receiving optics photodetector responsivity and the transducer constant, at each detection wavelength, $O_{X}(R)$ is the overlap function profile at each detected wavelength, the exponential term takes into account the extinction along the propagation path of both the emitted $\left(\lambda_{0}\right)$ and Raman-shifted backscattered radiation $\left(\lambda_{X}\right)$, and $\partial \sigma_{X \text { eff }} / \partial \Omega$ is the effective differential Raman backscatter cross section of the species, which takes into account the frequency selection produced in the narrowband interference filters [16], [24] and is in general temperature-and hence altitude-dependent. In the case of the UPC lidar, this dependence can, however, be neglected (see Section III-A).

From (1) and (2)

$$
\begin{aligned}
\omega(R)=0.4853 & \times \frac{K_{N}}{K_{W}} \frac{O_{N}(R)}{O_{W}(R)} \frac{\frac{\partial \sigma_{\text {Neff }}}{\partial \Omega}}{\frac{\partial \sigma_{\text {eff }}}{\partial \Omega}} \frac{S_{W}(R)}{S_{N}(R)} \\
& \times \exp \left\{\int_{0}^{R}\left[\alpha\left(r, \lambda_{W}\right)-\alpha\left(r, \lambda_{N}\right)\right] d r\right\} .
\end{aligned}
$$

Differences in transmission at the Raman frequencies accounted for in the exponential term on the right of (3) above can be neglected in common situations. Thus, the Rayleigh contribution, which has been computed using atmospheric models, results in differences below $3 \%$ for altitudes where water vapor content is meaningful, while differences in aerosol extinction would only produce significant errors in heavily aerosol loaded atmospheres [typically, for aerosol optical depth (AOD) larger than 2] [14]. Although mere geometrical considerations indicate that the overlap functions ratio, $O_{N}(R) / O_{W}(R)$, of two channels sharing the same optical setup should be equal to unity independently of the particular implementation of the system, in real systems this might not be strictly true in the near range. Whiteman et al. [25] found for a typical system that this ratio was 1 only above $750 \mathrm{~m}$ and decreased to 0.94 at $300 \mathrm{~m}$. In this paper, we have used their errorless altitude as a security reference, and therefore we present WVMR measurements at altitudes greater than $750 \mathrm{~m}$, assuming identical overlap functions above that height. If accurate lower altitude water vapor measurements were required, a residual overlap correction function should be also determined and applied to the retrieved water vapor profiles [25], [26]. In our case, the WVMR can be, therefore, obtained by multiplying the ratio between the signal profiles corresponding to water vapor and nitrogen Raman channels by a calibration factor that has to be estimated for the particular lidar instrument. This factor depends on both the effective Raman backscatter cross sections and the system constants 
in each channel

$$
\omega(R)=F_{\mathrm{CAL}} \frac{S_{W}(R)}{S_{N}(R)}
$$

with

$$
F_{\mathrm{CAL}}=0.4853 \times \frac{\frac{\partial \sigma_{\text {Neff }}}{\partial \Omega}}{\frac{\partial \sigma_{W \text { eff }}}{\partial \Omega}} \frac{K_{N}}{K_{W}} .
$$

\section{Estimation OF THE CALIBRATION FACTOR}

The method applied in the UPC Raman lidar to determine the calibration factor above consists of determining both the effective cross-sectional ratio $\left(\partial \sigma_{N \text { eff }} / \partial \Omega\right) /\left(\partial \sigma_{W \text { eff }} / \partial \Omega\right)$ and the system constants ratio $K_{N} / K_{W}$ appearing in (5). For determining the former term, the effective differential Raman backscatter cross section of each channel is calculated as a summation of the spectral lines corresponding to the rotational-vibrational transitions, a frequency selected by the interference filter. [16]. Formally

$$
\frac{\partial \sigma_{X \mathrm{eff}}}{\partial \Omega}=\sum_{i} \frac{\partial \sigma_{X}\left(\lambda_{i}\right)}{\partial \Omega} t_{X}\left(\lambda_{i}\right)
$$

where $\lambda_{i}$ is the wavelength corresponding to each line of the Raman spectrum and $\partial \sigma_{X}\left(\lambda_{i}\right) / \partial \Omega$ and $t_{X}\left(\lambda_{i}\right)$ are, respectively, the differential backscatter cross section for the specie $X$ and the normalized transmission function of the corresponding interference filter at line wavelength $\lambda_{i}$.

The ratio between system constants $K_{N} / K_{W}$ needed to find the calibration factor in (5) can be determined from the measurement of diffuse sky radiation in the nitrogen and water vapor channels of the lidar instrument [17]. Once the "darkcurrent" offset-due to electronics and photodetector dark current in the case of an analog channel, or to dark counts in a photon-counting channel-has been removed from the detected signal, we obtain a background-radiation induced dc voltage $S_{B X}$ defined as

$$
S_{B X}=K_{X} A_{r} \Omega_{X} B_{X} L_{\lambda X}
$$

where $\Omega_{X}$ is the receiver field of view (sr), $L_{\lambda X}$ is the spectral radiance $\left(\mathrm{W} \cdot \mathrm{nm}^{-1} \cdot \mathrm{m}^{-2} \cdot \mathrm{sr}^{-1}\right)$ at the corresponding Ramanshifted wavelength, which is considered spectrally flat in the interference filters bandpass intervals, and $B_{X}$ can thus be identified as the interference filter effective bandwidth (nm) $\int t_{X}(\lambda) d \lambda$. Therefore,

$$
\frac{S_{B N}}{S_{B W}}=\frac{K_{N}}{K_{W}} \frac{\Omega_{N}}{\Omega_{W}} \frac{B_{N}}{B_{W}} \frac{L_{\lambda N}}{L_{\lambda W}} .
$$

Equation (8) shows that if the ratios between $\Omega_{X}, B_{X}$, and $L_{\lambda X}$ are known, the ratio between the system constants $K_{X}$, required in (5) for calculating the calibration factor $F_{\mathrm{CAL}}$, can be obtained by computing the ratio of the measured background-radiation induced offsets $S_{B X}$

$$
\frac{K_{N}}{K_{W}}=\frac{\Omega_{W}}{\Omega_{N}} \frac{B_{W}}{B_{N}} \frac{L_{\lambda W}}{L_{\lambda N}} \frac{S_{B N}}{S_{B W}} .
$$

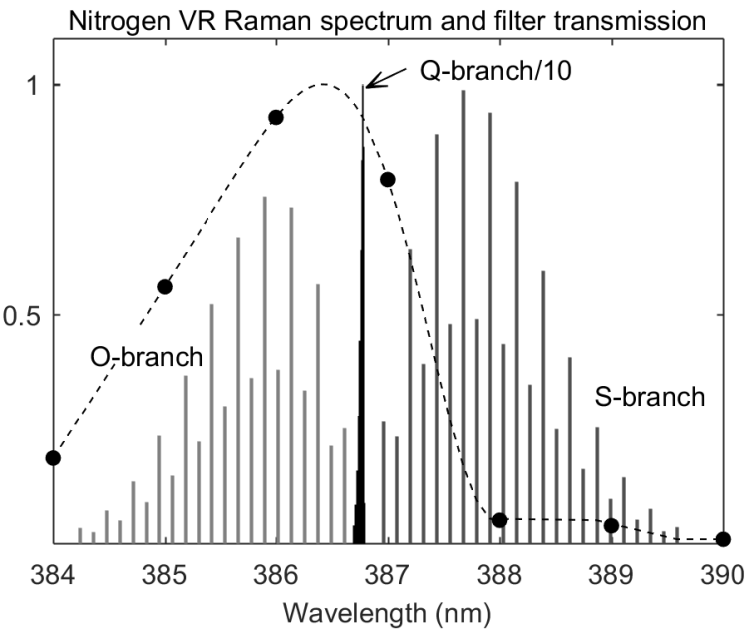

Fig. 1. Nitrogen vibro-rotational Raman spectrum (a.u.), calculated at 280-K, and the interpolated, normalized frequency response of the interference filter in the 387-nm channel (dashed line), where solid dots indicate filter data from the manufacturer.

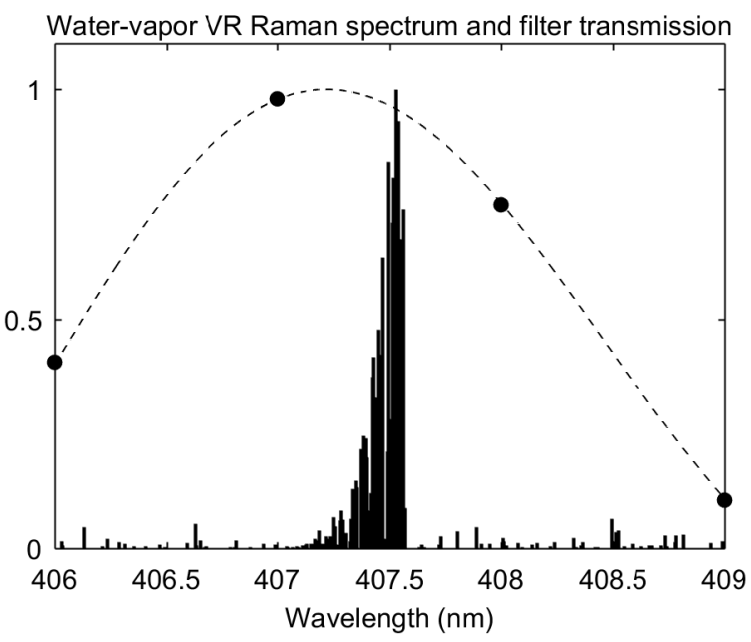

Fig. 2. Water vapor vibro-rotational Raman spectrum (a.u.), calculated at $280 \mathrm{~K}$, and the interpolated, normalized frequency response of the interference filter in the 407-nm channel (dashed line), where solid dots indicate filter data from the manufacturer.

\section{A. Determination of the Effective Cross-Sectional Ratio}

The filter frequency transmission functions used in (6) to calculate the effective differential Raman backscatter cross section of each channel have been provided by the filter manufacturer with a resolution of $1 \mathrm{~nm}$. A standard curve-fitting procedure has been applied to interpolate the transmission at each wavelength of the Raman spectra. The differential Raman cross sections for each spectral line have been computed following [20], [24] in the case of water vapor and [21] in the case of Nitrogen. Figs. 1 and 2 show, in arbitrary units, the distribution of the spectral lines for nitrogen and water vapor at $280 \mathrm{~K}$ and the normalized frequency response of the corresponding interference filters used in the UPC lidar.

In the case of UPC lidar, when a temperature of $280 \mathrm{~K}$ is considered, the effective differential Raman backscattered cross sections have resulted $2.40 \times 10^{-34} \mathrm{~m}^{2} / \mathrm{sr}$ for the nitrogen (computed between 384 and $390 \mathrm{~nm}$ ) and 
$7.03 \times 10^{-34} \mathrm{~m}^{2} / \mathrm{sr}$ for the water vapor (computed between 405 and $410 \mathrm{~nm}$ ). The ratio of the effective cross sections is thus 0.34 . However, the intensity of the rotational-vibrational Raman scattering spectral lines, and therefore also this ratio, depends on the temperature in the observed region. This makes the calibration factor being also temperature-and hence altitude-dependent [16], [17], [24]. This dependence, however, is strong for individual lines but not so much if the total backscatter is taken into account, resulting more significant if narrowband interference filters are used. In the case of the filters used in the UPC lidar, a temperature variation between 200 and $300 \mathrm{~K}$, which can be assumed as reasonable in the range of sensed altitudes, implies a difference in the effective cross-sectional ratio, and as a consequence in the calibration factor, of $2.3 \%$ in the worst case. The effective cross-sectional ratio used in the tests presented here has been calculated for a temperature of $280 \mathrm{~K}$, typical at low altitudes, and, therefore, this worst case will occur likely only in the upper troposphere, where water vapor signals are very weak and accurate measurements are anyway not possible. Another source of uncertainty arises from the determination of the spectral response of the interference filters, which might be not easily measurable and can vary in time. Among other filter features, the effective cross sections are especially sensitive to variations of the response peak location [17]. When extreme shifts of $\pm 0.05 \mathrm{~nm}$ are considered in the response of the filters used in the UPC lidar, the calculated error in the effective cross-sectional ratio results to be, respectively, $\sim+5 \%$ and $-2 \%$. Both estimated errors-the ones related, respectively, to the temperature and the filter peak-frequency uncertainties-will be included in the error bars calculation of the water vapor measurements.

\section{B. Determination of the System Constants Ratio}

Regarding the computation, following (9), of the system constants ratio, we have first assumed as an approximation that the field of view in both channels is approximately the same since the receiving optics defining the receiver field of view is common to both channels [18] and given the proximity of the corresponding wavelengths. Regarding the data provided by the manufacturer of the filters, it has been also assumed that the effective bandwidths ratio $B_{W} / B_{N}$ is 0.91 . The ratio between the sky spectral radiances at a given observation angle $L_{\lambda W} / L_{\lambda N}$ depends on the location, the day of the year, the time of the day, the aerosol loading, and the meteorological conditions. This ratio can be estimated for each individual calibration using the radiative transfer model GAME [19] and auxiliary measurements (lidar, sun photometer, and meteorological variables) providing input parameters for the model. GAME accounts for the scattering and absorption processes due to gases and aerosols. Gaseous absorption $\left(\mathrm{H}_{2} \mathrm{O}, \mathrm{CO}_{2}\right.$, $\mathrm{O}_{2}$, and $\mathrm{O}_{3}$ ) is treated with the correlated $k$ distribution, using a line by line code [27] and multiple scattering effects are treated using the discrete ordinates method [28]. GAME allows accurate treatment of scattering and absorption by aerosols and molecules [29]. Calibrations were performed in cloudfree conditions for accurate radiance simulations. Figs. 3 and 4 illustrate the influence of the day of the year, the time during

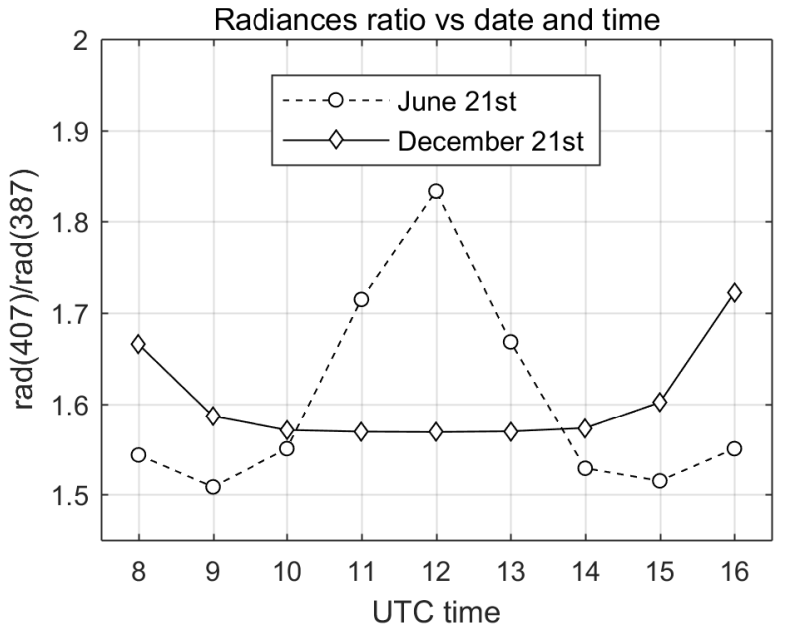

Fig. 3. Sky radiances ratio at different dates and time. For these simulations, the rest of input parameters $[\alpha(R)$, sun photometer data, and meteorological variables] have been kept constant.

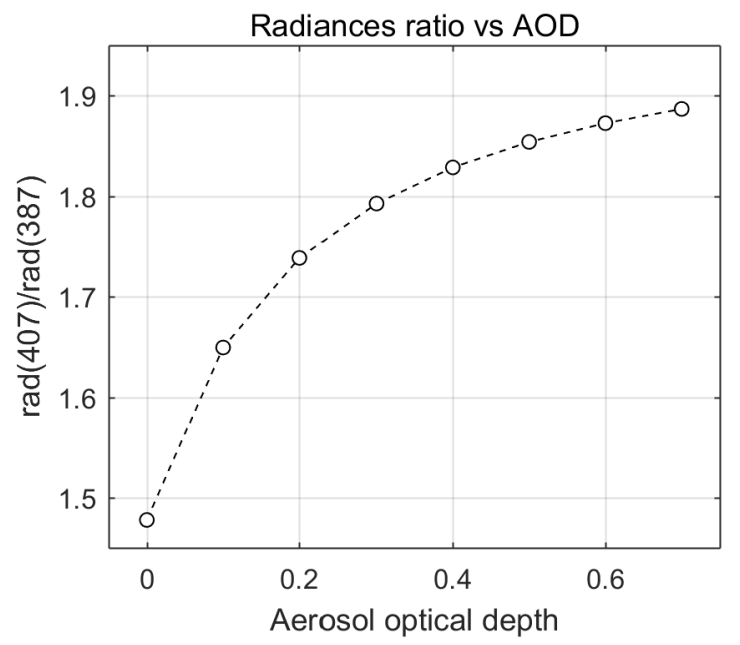

Fig. 4. Sky radiances ratio for different values of the AOD. These simulations use a fixed date and time (July 24 at 12:00 UTC) and the same aerosol vertical distribution scaled to match the observed AOD.

the day, and the aerosol loading conditions in the estimation of the spectral radiances ratio. Fig. 3 shows the diurnal evolution of $L_{\lambda W} / L_{\lambda N}$ at the two solstices for a fixed AOD of 0.15 and the same predefined aerosol vertical distribution, while Fig. 4 shows the variation of $L_{\lambda W} / L_{\lambda N}$ as a function of AOD for a given day, time, and aerosol vertical distribution. One sees that at the coordinates of Barcelona, $L_{\lambda W} / L_{\lambda N}$ can vary, in the same day (summer solstice), between 1.51 and $1.83(21 \%)$ depending on the time of the day. In turn, if the simulations are performed for the same time (12:00), variations of the radiances ratio can range between 1.57 and $1.83(17 \%)$ depending on the day of the year. Another interesting result is the influence of the aerosols, which tend to increase the ratio $L_{\lambda W} / L_{\lambda N}$. If we take an AOD of 0.2 , the increase of $L_{\lambda W} / L_{\lambda N}$ with respect to a situation without aerosols $(\mathrm{AOD}=0)$ and with the assumed aerosol vertical distribution is $18 \%$. These calculations show the importance of accurate simulations of the radiances when determining the calibration factor. 


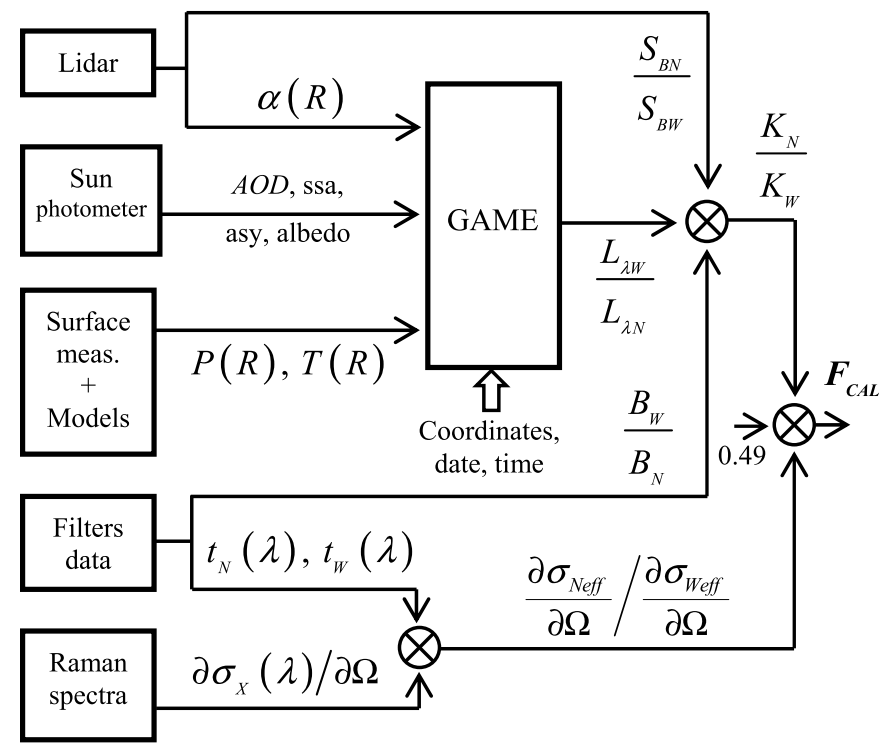

Fig. 5. Scheme showing the calibration procedure.

Finally, the background-induced signals, $S_{B N}$ and $S_{B W}$, can be obtained either directly from diffuse sky backgroundradiation measurements or from lidar signals at $25-30 \mathrm{~km}$, where the backscatter contribution can be assumed to be negligible. In practice, lidar signals are the preferred source because at the same time they provide the vertical aerosol structure required as input in the radiative transfer model.

Fig. 5 shows schematically the calibration procedure described above. Sun photometer data, obtained from aerosol robotic network (AERONET; http://aeronet.gsfc.nasa.gov/) [30], provide inputs $[\mathrm{AOD}$, albedo, single scattering albedo, and the asymmetry factor (asy)] for GAME. A lidar measurement provides both the sky background signals, $S_{B N}$ and $S_{B W}$, and, after proper processing, the extinction profile, $\alpha(R)$, used as a qualitative information by GAME to distribute the column-integrated AERONET parameters in layers. In our case, the extinction profiles for daytime measurements are obtained performing the Klett-Ferland's algorithm [31], using sun photometer data to constrain the AOD and determine the required lidar ratio. Overlap functions are periodically assessed from nighttime Raman measurements using the procedure proposed by Wandinger and Ansmann [32], and used to correct the lidar signals for both obtaining the extinction profile in the lowest altitudes and minimizing bias errors. Not having accurate information about the vertical distribution of the aerosols in the boundary layer would imply an additional uncertainty in the calibration of $1 \%$. This induced error has been empirically estimated by using a large enough number of different profiles (corresponding to different dates) and computing the standard deviation (SD) of the simulated radiances ratio when the rest of input parameters remain constant. Profiles of some meteorological variables, namely, pressure $P(R)$ and temperature $T(R)$, which can be obtained from daily radiosoundings at 12:00 AM or by using atmospheric models and surface measurements, are also used as input in GAME. Both alternatives have been tested and no significant differences have been observed $(<0.3 \%$ in all the cases). Atmospheric models plus

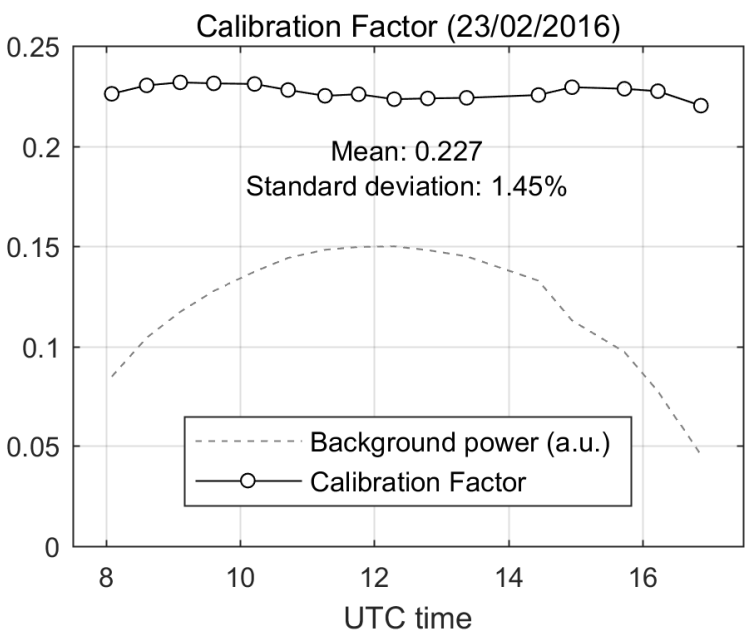

(a)

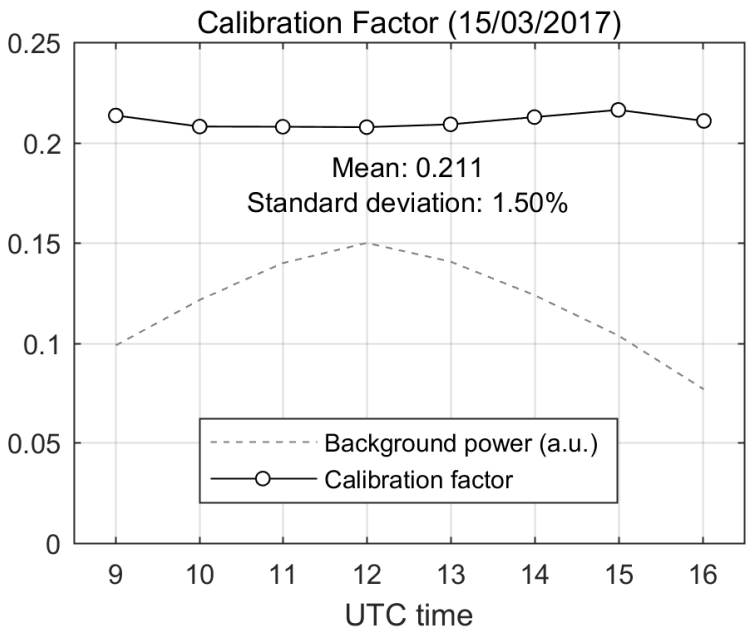

(b)

Fig. 6. Sets of multiple calibrations from sunrise to sunset performed (a) February 23, 2016 and (b) March 15, 2017. The detected signal at 387-nm channel (a.u.) is also plotted as a proxy of the variations of the diffuse background radiation.

local surface measurements have been finally chosen in the tests presented in this paper. This choice states the calibration method as not dependent on radiosondes launchings, what permits performing calibrations at any time during the day. An accurate knowledge of the frequency response of the interference filters of the nitrogen and water vapor Raman channels, $t_{N}(\lambda)$ and $t_{W}(\lambda)$, is also needed for determining the bandwidth ratio $\left(B_{W} / B_{N}\right)$ in (9) and, as well as the differential cross-sectional Raman spectra, for calculating the effective Raman cross sections, as described in Section III.

\section{RESULTS}

A set of calibration measurements during 2016 and 2017 has been used to obtain the ratio of background photon-counting mode signals in the lidar instrument. Simulations with GAME to determine in each case the ratio of spectral radiances have also been performed. With these results and with the rest of estimated parameters, the calibration factor $F_{\mathrm{CAL}}$ has been then calculated using (5) and (10). Fig. 6 shows, as examples, the resulting values for two sets of multiple calibrations performed in (a) February 23, 2016 between 8:00 UTC and 
TABLE I

Results of CALibrations Between FEBRUARY 2016 AND MARCH 2017

\begin{tabular}{cccc}
\hline \hline Date & $n_{C A L}$ & $F_{C A L}$ & $S D$ \\
\hline $2016 / 02 / 22$ & 13 & 0.230 & $1.11 \%$ \\
$2016 / 02 / 23$ & 16 & 0.227 & $1.45 \%$ \\
$2016 / 04 / 11$ & 1 & 0.208 & - \\
$2016 / 04 / 13$ & 1 & 0.207 & - \\
$2016 / 04 / 18$ & 1 & 0.205 & - \\
$2016 / 05 / 03$ & 1 & 0.205 & - \\
$2016 / 06 / 20$ & 1 & 0.206 & - \\
$2016 / 06 / 23$ & 9 & 0.209 & $1.47 \%$ \\
$2017 / 02 / 17$ & 1 & 0.212 & - \\
$2017 / 02 / 21$ & 1 & 0.214 & - \\
$2017 / 03 / 07$ & 1 & 0.213 & - \\
$2017 / 03 / 15$ & 9 & 0.211 & $1.50 \%$ \\
$2017 / 03 / 17$ & 6 & 0.208 & $0.82 \%$ \\
$2017 / 04 / 04$ & 7 & 0.198 & $1.39 \%$ \\
\hline \hline
\end{tabular}

$N$ : Number of calibrations during the day; $S D$ : standard deviation if multiple calibrations available.

17:30 UTC and in (b) March 15, 2017 between 9:00 and 17:00. The corresponding background lidar signal at $25-30 \mathrm{~km}$ from the $387-\mathrm{nm}$ channel, used as a proxy for the diffuse sky background condition, is also plotted to illustrate the variations of the incident radiation during the calibrations. Small variations of the calibration factor are visible but without any significant tendency, which indicates that the obtained calibration factor is independent of the solar zenith angle and of the amount of sky radiation.

Other calibrations, some of them made of multiple measurements during the same day, and others single measurements, have been performed between February 2016 and March 2017. The results, in terms of daily average, are shown in Table I, where $n_{\mathrm{CAL}}$ is the number of calibrations made on the same day and $\mathrm{SD}$ is the $\mathrm{SD}$ of the estimated calibration factor, $F_{\mathrm{CAL}}$ (when $n_{\mathrm{CAL}}>1$ ).

On the one hand, results show that the stability of the calibration factor is very good for relatively long periods of time (the relative SD during 11 dates of calibrations ranging almost a year, between April 11, 2016 and May 17, 2017, is only $1.52 \%)$. On the other hand, more significant changes can also occur (e.g., $\sim 10 \%$ between February 23, 2016 and April 11, 2016 and 5\% between May 17, 2017 and April 4, 2017). These variations, which arise from systematic changes in the instrument conditions such as uneven efficiency reduction (aging) in the photodetectors or variations in the frequency response of the filters, most of the times are not easily predictable and reveal the need of frequent periodic calibrations [33]. For this reason, having at our disposal a convenient, reliable calibration method presents great advantages.

\section{Water VAPor Mixing Ratio Profiles COMPARISON WITH RADIOSONDE DATA}

In this section, several WVMR profiles obtained from lidar measurements and the corresponding estimated calibration factor are presented. These profiles are compared with the ones calculated from radiosonde data [the air temperature, $T(R)$, the air pressure, $p(R)$, and the relative humidity, $U_{w}(R)$ ] [14]. The WVMR, $\omega(R)$, is related to the air pressure, which is supplied by the radiosonde and the water vapor pressure, $e(R)$

$$
\omega(R)=0.622 \frac{e(R)}{p(R)-e(R)} .
$$

In turn, the water vapor pressure can be expressed as a function of the relative humidity (over water), $U_{w}(R)$, which is a parameter provided by the radiosonde, and the saturation pressure, $e_{w}(R)$

$$
e(R)=U_{w}(R) e_{w}(R) .
$$

Finally, the saturation pressure depends on the air temperature

$$
\begin{aligned}
e_{w}(R) & =6.107 \exp \left\{\frac{M_{A}[T(R)-273]}{M_{B}+[T(R)-273]}\right\} \\
T & <273 \mathrm{~K} \rightarrow M_{A}=17.84, \quad M_{B}=254.4 \\
T & >273 \mathrm{~K} \rightarrow M_{A}=17.08, \quad M_{B}=234.2 .
\end{aligned}
$$

Fig. 7 shows four cases in which water vapor profiles from lidar measurements and from radiosonde data are plotted. In all the cases, lidar retrievals have been obtained from 150 min measurements with spatial smoothing of $150 \mathrm{~m}$ below $3 \mathrm{~km}$ and $300 \mathrm{~m}$ above. The uncertainty in the lidar retrievals, $\sigma_{\omega}$, represented in the plots of Fig. 7 by error bars, has been calculated applying the error-propagation classic formulation [34] to (4)

$$
\begin{aligned}
\sigma_{\omega}^{2} & =\left(\frac{\partial \omega}{\partial F_{\mathrm{CAL}}}\right)^{2} \sigma_{F_{\mathrm{CAL}}}^{2}+\left(\frac{\partial \omega}{\partial S_{W}}\right)^{2} \sigma_{S_{W}}^{2}+\left(\frac{\partial \omega}{\partial S_{N}}\right)^{2} \sigma_{S_{N}}^{2} \\
& =\left(\frac{S_{W}}{S_{N}}\right)^{2} \sigma_{F_{\mathrm{CAL}}}^{2}+\left(\frac{F_{\mathrm{CAL}}}{S_{N}}\right)^{2} \sigma_{S_{W}}^{2}+\left(\frac{F_{\mathrm{CAL}} S_{W}}{S_{N}^{2}}\right)^{2} \sigma_{S_{N}}^{2}
\end{aligned}
$$

where $\sigma_{S_{N}}^{2}$ and $\sigma_{S_{W}}^{2}$ are the noise variances of both lidar signals at each retrieval altitude and $\sigma_{F_{\mathrm{CAL}}}$ is a statistical error assigned to the calibration factor: $2.3 \%$ corresponding to the temperature uncertainty and $5 \%$ to the filter location uncertainty (both described in Section III-A). Considering that they are independent sources of error the composite uncertainty assigned to the calibration factor results $\sigma_{F_{\mathrm{CAL}}}=5.5 \%$.

In general, there is a good agreement between profiles regarding rough vertical structural features. Table II shows the mean bias and the relative SD between lidar retrievals and radiosonde data, calculated at altitudes where WVMR is greater than one, for a set of 14 cases between December 2015 and May 2017. The calibration factor used in each lidar retrieval has been the closest available to the date of the measurements. The mean bias is, in eight of the cases, below 3\%, in four cases between 5\% and $10 \%$ and in two cases significantly bigger (14\% and $25 \%)$. The relative SD is in turn below $10 \%$ in three cases, between $10 \%$ and $20 \%$ in nine of the cases and above $30 \%$ for the two cases with the largest biases.

These differences arise mainly from fine details in the profiles that can be partly explained by the lack of both spatial and temporal exact coincidence between lidar and radiosonde measurements. First of all, the lidar and the radiosonde launch 


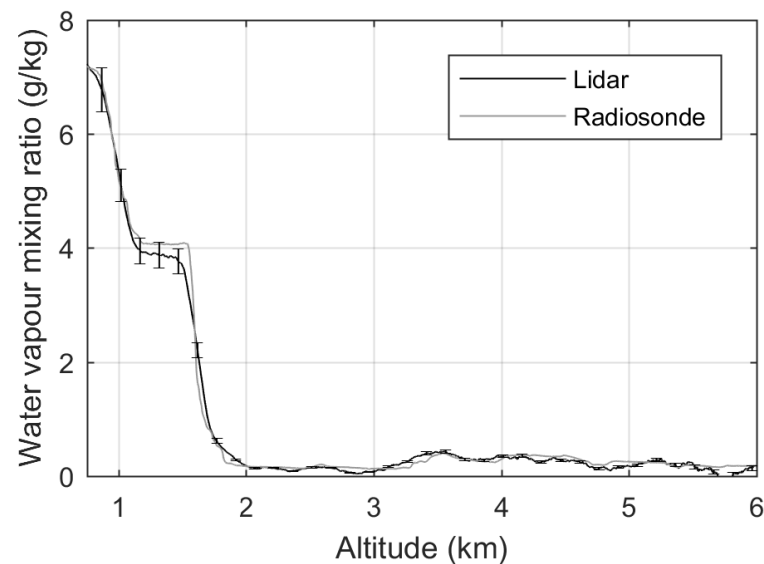

(a)

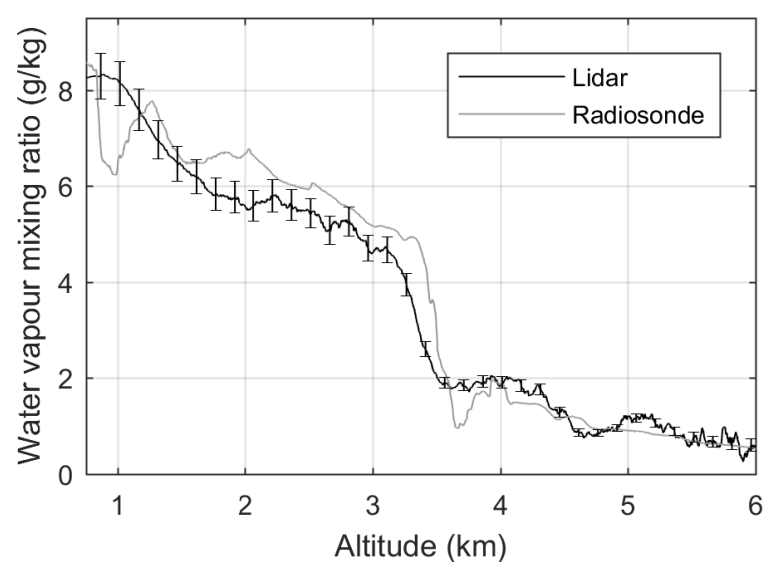

(c)

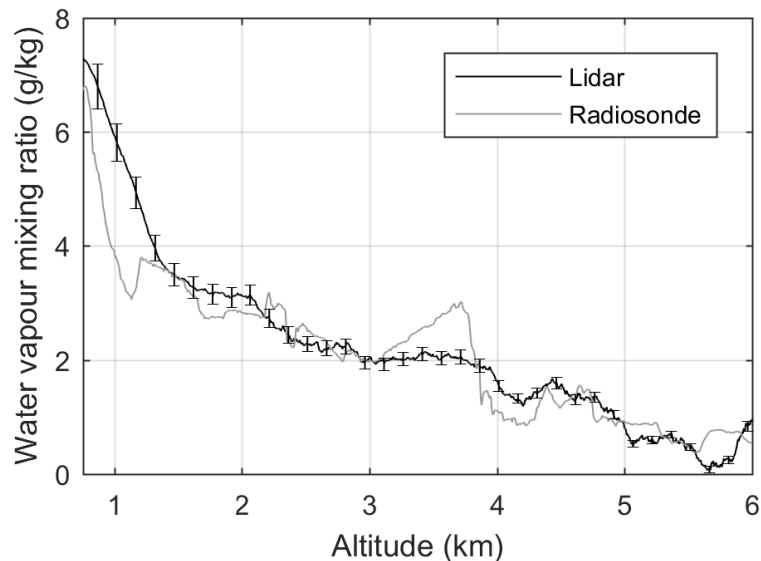

(b)

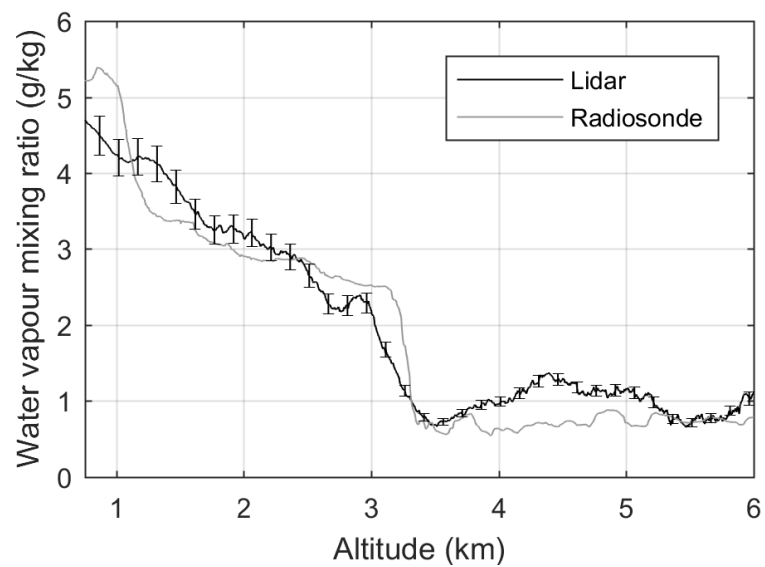

(d)

Fig. 7. Four cases of lidar WVMR retrievals along with almost co-located, almost simultaneous radiosonde measurements. (a) December 11,2015 at 00:46. (b) January 28, 2016 at 00:45. (c) September 17, 2016 at 00:45. (d) November 11, 2016 at 00:46. The calibration factor used in cases (a) and (b) is $F_{\mathrm{CAL}}=$ 0.230 (calibrated February 22, 2016); for case (c) $F_{\mathrm{CAL}}=0.209$ (calibrated June 23, 2016); and for case (d) $F_{\mathrm{CAL}}=0.212$ (calibrated February 17,2017 ).

TABLE II

Mean Bias and Relative SD Between Lidar Retrievals AND RADIOSONDE DATA AT ALTITUDES WITH WVMR > 1

\begin{tabular}{lcc}
\hline \hline \multicolumn{1}{c}{ Date } & Bias (\%) & SD (\%) \\
\hline $2015 / 12 / 04$ & 2.50 & 11.50 \\
$2015 / 12 / 11^{*}$ & -0.77 & 7.49 \\
$2016 / 01 / 12$ & 1.88 & 9.20 \\
$2016 / 01 / 21$ & 25.65 & 30.87 \\
$2016 / 01 / 28^{*}$ & -6.72 & 17.47 \\
$2016 / 05 / 28$ & -13.78 & 35.18 \\
$2016 / 06 / 20$ & -0.71 & 3.77 \\
$2016 / 07 / 06$ & -2.13 & 17.12 \\
$2016 / 09 / 17 *$ & 6.55 & 12.47 \\
$2016 / 11 / 11^{*}$ & 2.77 & 12.40 \\
$2017 / 01 / 14$ & -8.75 & 14.86 \\
$2017 / 04 / 19$ & 1.71 & 10.89 \\
$2017 / 04 / 23$ & -9.47 & 13.15 \\
$2017 / 05 / 25$ & -2.05 & 15.53 \\
\hline \hline
\end{tabular}

* Cases shown in Fig. 7

site are separated $\sim 700 \mathrm{~m}$. Then, the radiosonde trajectory during its ascent, which is different in each case, results into horizontally projected distances between the radiosonde and the lidar line of sight that could eventually become significant.
TABLE III

DRIFT DistanCES OF RAdIOSONDES AT 2- AND 6-km Altitude

\begin{tabular}{ccc}
\hline \hline Date - Time & Distance at $2 \mathrm{~km}$ & Distance at $6 \mathrm{~km}$ \\
\hline $2015 / 12 / 11-00: 46$ & 1.67 & 4.87 \\
$2016 / 01 / 28-00: 45$ & 3.87 & 13.2 \\
$2016 / 09 / 17-00: 46$ & 2.98 & 10.88 \\
$2016 / 11 / 11-00: 45$ & 3.48 & 14.19 \\
\hline \hline
\end{tabular}

In particular, the horizontal drifts at 2 and $6 \mathrm{~km}$ altitude for the cases presented in Fig. 5 are given in Table III. The location of the lidar station (in a urban environment, at the bottom of a 500-m altitude mountain range, and at a distance of $5 \mathrm{~km}$ from the sea) as well as the complex vertical structure usually observed over Barcelona [35] let us think that radiosonde drifts can significantly impact water vapor atmospheric measurements validation.

Besides, temporal coincidence between lidar and radiosonde measurements is not either perfect. Thus, radiosonde launchings take place every night at 00:00 UTC, and they provide instantaneous values for each altitude level, reaching $10-\mathrm{km}$ altitude in $30-35 \mathrm{~min}$. The lidar profiles presented here were synchronized with the overpasses of the CloudAerosol Lidar and Infrared Pathfinder Satellite [36], starting 


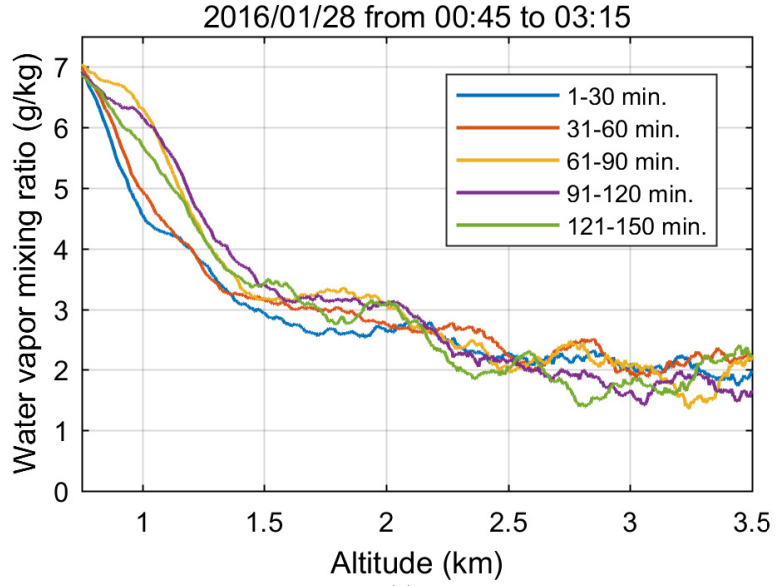

(a)

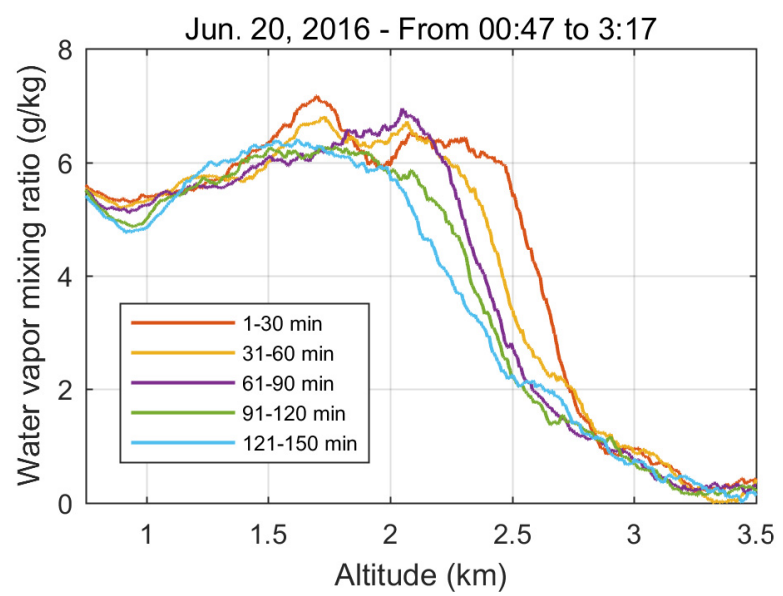

(b)

Fig. 8. Lidar retrievals of the WVMR averaged every 30 consecutive minutes in (a) January 28, 2016, between 00:45 and 03:15 UTC and (b) June 20, 2016, from 00:47 to $03: 17$.

at 00:45 UTC and accumulating acquisitions during $150 \mathrm{~min}$. They correspond, thus, to averaged measurements between 00:45 and 03:15 UTC. Apart from the temporal noncoincidence between measurements, the water vapor vertical structure usually changes during a 150-min lidar measurement, as can be observed in Fig. 8, where two WVMR profiles are split into 30-min retrievals.

The considerations previously mentioned show the difficulties and uncertainties that, in our case, radiosondes involve when they are used as the reference instrument for validation/ calibration of water vapor measurements.

\section{CONCLUSION}

The calibration factor for water vapor retrievals has been estimated from the characterization of Raman cross sections and system constants at both water vapor and Nitrogen Raman wavelengths. Raman cross sections have been obtained from Raman scattering models and interference filter parameters. Whereas system constants determination requires far-range background-level measurements, interference filters parameters, and auxiliary data (lidar products, sun photometer measurements, and atmospheric variables profiles) to be input of a radiative transfer model for estimating incident radiance levels.
Multiple calibrations carried out during the same day, from sunrise to sunset, have yielded that the method is virtually independent of the illumination intensity or the solar zenith angle. In turn, regular calibrations of the UPC lidar over a yearlong have shown that the calibration factor, although remaining approximately constant for relatively long periods, can suffer sudden, unpredictable variations mainly due to systematic changes in the instrument conditions, which makes indispensable frequent regular calibrations of the water vapor channel.

Comparisons between lidar WVMR profiles using the calibration factor obtained with this method and data from reference radiosondes are in good agreement when rough features are assessed. However, differences in the finer details of the vertical water vapor profiles (bias about 5\% and relative SD between $10 \%$ and $15 \%$ in most of the cases), reveal the key uncertainties associated with the use of radiosondes, namely, horizontal drifts, and, up to a point, temporal variations (Section V). Alternatively, the method proposed here overcomes these problems by relying on simultaneous reference lidar, sun photometer, and surface measurements.

\section{REFERENCES}

[1] I. M. Held and B. J. Soden, "Water vapor feedback and global warming," Аnпu. Rev. Energy Environ., vol. 25, no. 1, pp. 441-475, Nov. 2000.

[2] J. Reichardt, U. Wandinger, V. Klein, I. Mattis, B. Hilber, and R. Begbie, "RAMSES: German Meteorological Service autonomous Raman lidar for water vapor, temperature, aerosol, and cloud measurements," Appl. Opt., vol. 51, no. 34, pp. 8111-8131, 2012.

[3] G. Vaughan, D. P. Wareing, L. Thomas, and V. Mitev, "Humidity measurements in the free troposphere using Raman backscatter," Quart. J. Roy. Meteorol. Soc., vol. 114, no. 484, pp. 1471-1484, 1988.

[4] D. Scheiben, A. Schanz, B. Tschanz, and N. Kämpfer, "Diurnal variations in middle-atmospheric water vapor by ground-based microwave radiometry," Atmos. Chem. Phys., vol. 13, no. 14, pp. 6877-6886, 2013.

[5] D. D. Turner, S. A. Clough, J. C. Liljegren, E. E. Clothiaux, K. E. Cady-Pereira, and K. L. Gaustad, "Retrieving liquid water path and precipitable water vapor from the Atmospheric Radiation Measurement (ARM) microwave radiometers," IEEE Trans. Geosci. Remote Sens., vol. 45, no. 11, pp. 3680-3689, Nov. 2007.

[6] M. D. Alexandrov et al., "Columnar water vapor retrievals from multifilter rotating shadowband radiometer data," J. Geophys. Res. Atmos., vol. 114, no. D2, p. D02306, 2009.

[7] D. Pérez-Ramírez, F. Navas-Guzmán, H. Lyamani, J. Fernández-Gálvez, F. J. Olmo, and L. Alados-Arboledas, "Retrievals of precipitable water vapor using star photometry: Assessment with Raman lidar and link to sun photometry," J. Geophys. Res. Atmos., vol. 117, no. 5, p. D05202, Mar. 2012.

[8] T. M. Weckwerth, K. J. Weber, D. D. Turner, and S. M. Spuler, "Validation of a water vapor micropulse differential absorption lidar (DIAL)," J. Atmos. Ocean. Technol., vol. 33, no. 11, pp. 2353-2372, Sep. 2016.

[9] D. D. Turner and U. Löhnert, "Information content and uncertainties in thermodynamic profiles and liquid cloud properties retrieved from the ground-based Atmospheric Emitted Radiance Interferometer (AERI)," J. Appl. Meteorol. Climatol., vol. 53, no. 3, pp. 752-771, Dec. 2013.

[10] M. Bevis, S. Businger, T. A. Herring, C. Rocken, R. A. Anthes, and R. H. Ware, "GPS meteorology: Remote sensing of atmospheric water vapor using the global positioning system," J. Geophys. Res., vol. 97, no. D14, p. 15787 , Oct. 1992.

[11] D. N. Whiteman, S. H. Melfi, and R. A. Ferrare, "Raman lidar system for the measurement of water vapor and aerosols in the Earth's atmosphere," Appl. Opt., vol. 31, no. 16, pp. 3068-3082, Jun. 1992.

[12] T. Leblanc, I. S. McDermid, and T. D. Walsh, "Ground-based water vapor Raman lidar measurements up to the upper troposphere and lower stratosphere for long-term monitoring," Atmos. Meas. Techn., vol. 5, no. 1, pp. 17-36, 2012. 
[13] F. Navas-Guzmán, J. Fernández-Gálvez, M. J. Granados-Muñoz, J. L. Guerrero-Rascado, J. A. Bravo-Aranda, and L. Alados-Arboledas, "Tropospheric water vapour and relative humidity profiles from lidar and microwave radiometry," Atmos. Meas. Techn., vol. 7, no. 5, pp. 1201-1211, 2014.

[14] I. Mattis et al., "Relative-humidity profiling in the troposphere with a Raman lidar," Appl. Opt., vol. 41, no. 30, pp. 6451-6462, 2002.

[15] E. Landulfo, R. F. Da Costa, A. S. Torres, F. J. S. Lopes, D. N. Whiteman, and D. D. Venable, "Raman water vapor lidar calibration," Proc. SPIE, vol. 7479, pp. 74790J-1-74790J-9, Oct. 2009.

[16] D. D. Venable, D. N. Whiteman, M. N. Calhoun, A. O. Dirisu, R. M. Connell, and E. Landulfo, "Lamp mapping technique for independent determination of the water vapor mixing ratio calibration factor for a Raman lidar system," Appl. Opt., vol. 50, no. 23, pp. 4622-4632, 2011.

[17] V. Sherlock, A. Hauchecorne, and J. Lenoble, "Methodology for the independent calibration of Raman backscatter water-vapor lidar systems," Appl. Opt., vol. 38, no. 27, pp. 5816-5837, 1999.

[18] D. Kumar et al., "Six-channel polychromator design and implementation for the UPC elastic/Raman lidar," Proc. SPIE, vol. 8182, pp. 81820W-1-81820W-10, Sep. 2011.

[19] P. Dubuisson, D. Dessailly, M. Vesperini, and R. Frouin, "Water vapor retrieval over ocean using near-infrared radiometry," J. Geophys. Res., vol. 109, no. D19, p. D19106, Oct. 2004.

[20] G. Avila, J. M. Fernández, G. Tejeda, and S. Montero, "The Raman spectra and cross-sections of $\mathrm{H}_{2} \mathrm{O}, \mathrm{D}_{2} \mathrm{O}$, and $\mathrm{HDO}$ in the $\mathrm{OH} / \mathrm{OD}$ stretching regions," J. Mol. Spectrosc., vol. 228, no. 1, pp. 38-65, 2004.

[21] F. Liu and F. Yi, "Lidar-measured atmospheric $\mathrm{N}_{2}$ vibrational-rotational Raman spectra and consequent temperature retrieval," Opt. Express, vol. 22, no. 23, pp. 27833-27844, 2014.

[22] U. Wandinger, "Raman lidar," in Lidar: Range-Resolved Optical Remote Sensing of the Atmosphere, C. Weitkamp, Ed. New York, NY, USA: Springer, 2005, pp. 241-272.

[23] R. M. Measures, Laser Remote Sensing: Fundamentals and Applications. Malabar, FL, USA: Krieger, 1992.

[24] M. Adam, "Notes on temperature-dependent lidar equations," J. Atmos. Ocean. Technol., vol. 26, no. 6, pp. 1021-1039, 2009.

[25] D. N. Whiteman et al., "Raman lidar measurements during the international $\mathrm{H}_{2} \mathrm{O}$ project. Part I: Instrumentation and analysis techniques," $J$. Atmos. Ocean. Technol., vol. 23, no. 2, pp. 157-169, Feb. 2006.

[26] M. Walker, D. Venable, D. N. Whiteman, and T. Sakai, "Application of the lamp mapping technique for overlap function for Raman lidar systems," Appl. Opt., vol. 55, no. 10, pp. 2551-2558, 2016.

[27] P. Dubuisson, J. C. Buriez, and Y. Fouquart, "High spectral resolution solar radiative transfer in absorbing and scattering media: Application to the satellite simulation," J. Quant. Spectrosc. Radiat. Transf., vol. 55, no. 1, pp. 103-126, 1996

[28] K. Stamnes, S.-C. Tsay, W. Wiscombe, and K. Jayaweera, "Numerically stable algorithm for discrete-ordinate-method radiative transfer in multiple scattering and emitting layered media," Appl. Opt., vol. 27, no. 12, pp. 2502-2509, 1988.

[29] R. Barragan, S. Romano, M. Sicard, P. Burlizzi, M. R. Perrone, and A. Comeron, "Estimation of mineral dust direct radiative forcing at the European Aerosol Research Lidar NETwork site of Lecce, Italy, during the ChArMEx/ADRIMED summer 2013 campaign: Impact of radiative transfer model spectral resolutions," J. Geophys. Res. Atmos., vol. 121, no. 17, pp. 10237-10261, 2016.

[30] B. N. Holben et al., "AERONET—A federated instrument network and data archive for aerosol characterization," Remote Sens. Environ., vol. 66 , no. 1, pp. 1-16, Oct. 1998.

[31] J. D. Klett, "Lidar inversion with variable backscatter/extinction ratios," Appl. Opt., vol. 24, no. 11, pp. 1638-1643, Jun. 1985.

[32] U. Wandinger and A. Ansmann, "Experimental determination of the lidar overlap profile with Raman lidar," Appl. Opt., vol. 41, no. 3, pp. 511-514, Jan. 2002

[33] G. Martucci, J. Voirin, V. Simeonov, L. Renaud, and A. Haefele, "A novel automatic calibration system for water vapor Raman LIDAR," in Proc. EPJ Web Conf., vol. 176, 2018, p. 05008.

[34] H. H. Ku, "Notes on the use of propagation of error formulas," J. Res. Nat. Bureau Standards C, Eng. Instrum., vol. 70C, no. 4, pp. 263-273, 1966.

[35] M. Sicard, C. Pérez, F. Rocadenbosch, J. M. Baldasano, and D. García-Vizcaino, "Mixed-layer depth determination in the Barcelona coastal area from regular lidar measurements: Methods, results and limitations," Boundary-Layer Meteorol., vol. 119, no. 1, pp. 135-157, 2006.
[36] D. M. Winker, J. R. Pelon, and M. P. McCormick, "CALIPSO mission: Spaceborne lidar for observation of aerosols and clouds," Proc. SPIE, vol. 4893, pp. 4811-4893, Mar. 2003.

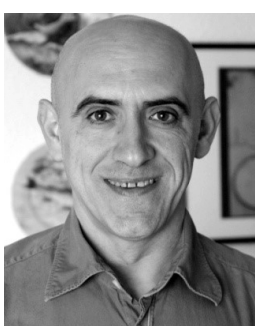

Constantino Muñoz-Porcar received the Electrical Engineering and $\mathrm{Ph} . \mathrm{D}$. degrees from the Technical University of Catalonia-BarcelonaTECH (UPC), Barcelona, Spain, in 1997 and 2012, recpectively.

$\mathrm{He}$ is currently an Associate Professor with the Department of Signal Theory and Communications. UPC. His research interests include atmospheric lidar systems for aerosols research, Doppler wind lidars and water vapor Raman lidars.

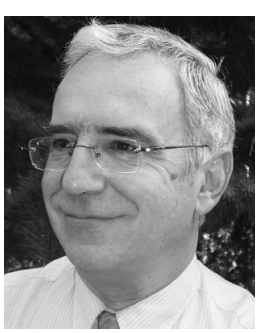

Adolfo Comerón received the Telecommunication Engineering degree from the Barcelona School of Telecommunication Engineering, Barcelona, Spain, in 1976, and the DEA and Dr.Eng. degrees from Paris-XI University, Orsay, France, in 1977 and 1980, respectively.

$\mathrm{He}$ is currently a Professor with the Technical University of Catalonia, Barcelona. His research interests include the study of nonlinear devices at IR wavelengths and the development of microwave and millimetre-wave receivers for satellite communication systems, free-space optical communications, lidar systems, remote detection, and sensing at optical wavelengths.

Dr. Comerón is a member of SPIE.

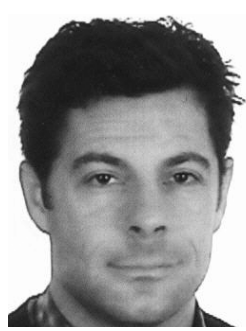

Michaël Sicard received the Ph.D. degree in physical methods for remote sensing from the Service d'Aéronomie, Institut Pierre-Simon-Laplace, Paris, France.

$\mathrm{He}$ is currently an Associate Professor with the Department of Signal Theory and Communications, University of Catalonia, Barcelona, Spain. His research interests include the development of lidar techniques and instruments for aerosol observations and the characterization of aerosol properties and their impact on the atmospheric radiative budget.

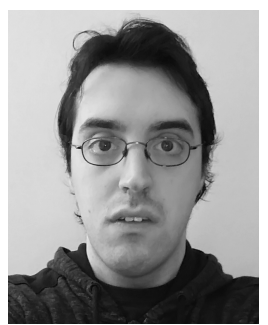

Ruben Barragan received the B.S. degree in environmental sciences from Rey Juan Carlos University, Madrid, Spain, in 2010, and the M.S. degree in remote sensing from the University of Alcalá de Henares, Alcalá de Henares, Spain, in 2011. $\mathrm{He}$ is currently pursuing the Ph.D. degree in telecom engineering (advisors, Dr. F. Rocadenbosch and Dr. M. Sicard) with the Universitat Politècnica de Catalunya, Barcelona, Spain.

His research interests include multispectral lidar signal processing, radiative forcing estimation, and characterization of precipitation by means of remote sensing technics. 


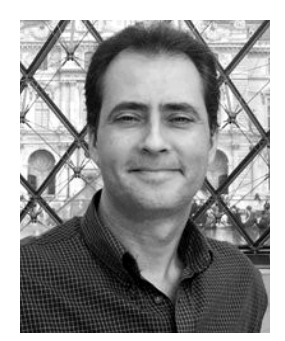

David Garcia-Vizcaino received the B.S. and Ph.D. degrees in telecommunications engineering from the Technical University of Catalonia (UPC), Barcelona, Spain, in 1998 and 2005, respectively.

Since 1996, he has been an Associate Professor with the Department of Signal Theory and Communications, UPC. His research interests include low-power coherent laser radar, laser Doppler anemometry systems, and atmospheric lidars for aerosol research.

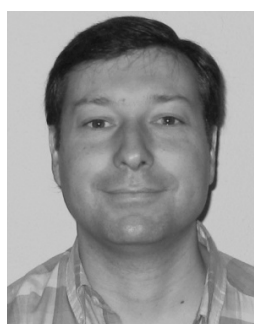

Alejandro Rodríguez-Gómez received the Telecommunication Engineering degree from the Technical University of Madrid, Madrid, Spain, in 1993, and the Ph.D. degree in telecommunication engineering from the Technical University of Catalonia (UPC), Barcelona, Spain, in 1998.

Since 1995, he has been an Associate Professor with the Department of Signal Theory and Communications, UPC. His research interests include low-power coherent and incoherent laser radar systems, atmospheric lidar systems, and free-space optical communications.

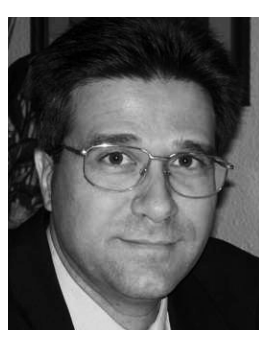

Francesc Rocadenbosch received the B.S. and $\mathrm{Ph} . \mathrm{D}$. degrees in telecommunications engineering from the Universitat Politècnica de Catalunya, Barcelona, Spain, in 1991 and 1996, respectively, and the M.B.A. degree from the University of Barcelona, Barcelona, in 2001.

$\mathrm{He}$ is currently an Associate Professor with the Technical University of Catalonia, Barcelona, where he is a Joint Representative with the Remote Sensing Laboratory. His research interests include lidar remote sensing and co-operative remote sensing sensors, related signal processing, and its application to off-shore wind farms (KIC-InnoEnergy strategic plan).

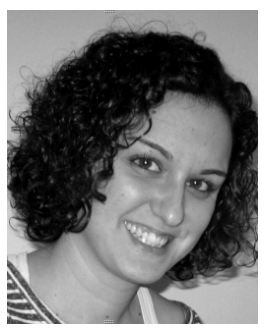

María José Granados-Muñoz received the B.Sc. degree in physics, the M.Sc. degree in geophysics and meteorology, and the Ph.D. degree in physics from the University of Granada, Granada, Spain, in 2009,2010 , and 2014, respectively.

She is currently a Post-Doctoral Researcher with the Remote Sensing Laboratory, Department of Signal Theory and Communications, Universitat Politècnica de Catalunya, Barcelona, Spain. Her research interests include atmospheric aerosol, solar radiation, and aerosol radiative forcing using combined active and passive remote sensing techniques. 